\title{
Protocols for Wireless Sensor Networks: A Survey
}

\author{
Aarti Kochhar ${ }^{1,2}$, Pardeep Kaur ${ }^{1}$, Preeti Singh ${ }^{1}$, and Sukesha Sharma ${ }^{1}$ \\ 1 University Institute of Engineering and Technology, Panjab University, Chandigarh, India \\ 2 Lovely Professional University, Phagwara, India
}

https://doi.org/10.26636/jtit.2018.117417

\begin{abstract}
This paper presents a survey on the MAC and network layer of Wireless Sensor Networks. Performance requirements of the MAC layer are explored. MAC layer protocols for battery-powered networks and energy harvestingbased networks are discussed and compared. A detailed discussion on design constraints and classification of routing protocols is presented. Several routing protocols are compared in terms of such parameters as: energy consumption, scalability, network lifetime and mobility. Problems that require future research are presented. The cross-layer approach for WSNs is also surveyed.
\end{abstract}

Keywords-cross layer, Medium Access Control, protocols, Wireless Sensor Networks.

\section{Introduction}

Any Wireless Sensor Network (WSN) application requires the physical environment to be sensed for data transmitted over a channel to a base station. Power is required in order to sense data and send it to the base station. It can be obtained from a battery or may be harvested from a natural source. One of the basic architectures of a sensor node is shown in Fig. 1 [1]. It comprises 4 units responsible for power, processing and communications. Most energy is consumed by processing and communications.

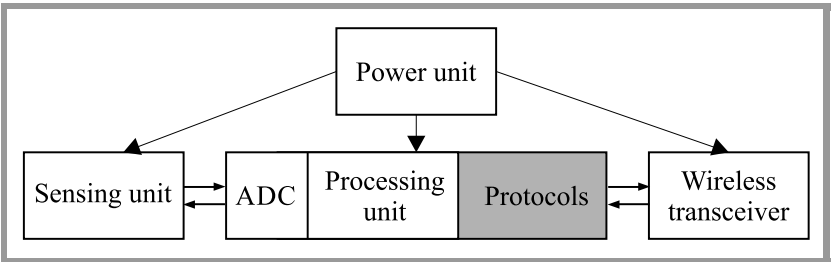

Fig. 1. Architecture of a typical sensor node.

In order to overcome energy, lifetime, traffic and mobility constraints, the communication protocol stack needs to be carefully designed.

The basic structure of a WSN protocol stack is discussed in Section 2. Transport and upper layers add reliability to the transmission of data only, which is not a key concern in the majority of WSN applications. Hence, only the data link layer (DLL) and the network layer are discussed in this paper. Energy consumption sources, classification, design constraints, respective protocols and outstanding research problems are discussed for the MAC and network layers. In Section 3, a sub-layer of DLL - Medium Access Control (MAC) layer - is surveyed. In Section 4, the network layer is examined. A comparison of both MAC and routing protocols has been tabulated in the respective sections. In Section 5, the cross layer approach, a technological advancement enhancing efficiency, is discussed.

\section{Protocol Stack in WSNs}

Proper design of the protocol stack is important for the overall efficiency of a WSN. WSN differs from conventional computer communication networks in the following ways:

- Contrary to computer network's well planned physical topology, the nodes are densely and randomly deployed in WSNs.

- Once designed, computer networks remain static, whereas WSNs are dynamic in nature. Failure of one node can change the entire topology. So, WSNs need to be self-configurable.

- Computer networks have IP addresses for their global identification. WSN nodes have no global identification because it creates a large overhead.

- Computer networks have a continuous supply of energy, whereas WSNs have limited resources. So, the WSN protocol stack needs to be energy-aware.

Protocol stacks in WSNs comprise five horizontal and five vertical levels. They have five layers and five management planes, as shown in Fig. 2 [2].

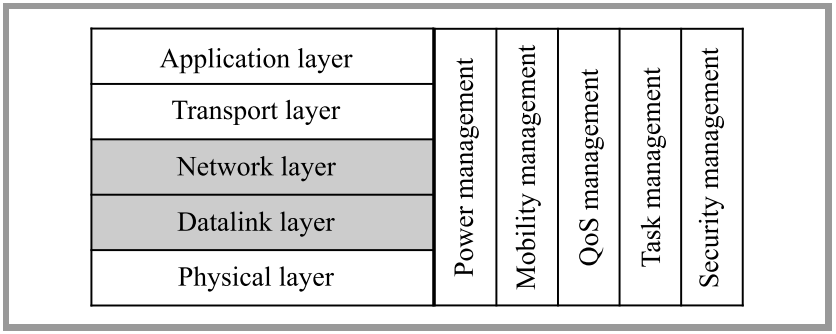

Fig. 2. Protocol stack of WSNs. 


\section{Data Link Layer}

DLL has two sub-layers: MAC and Logical Link Control (LLC). LLC is used for link management, flow and error control. MAC is responsible for assembling data into frames and for disassembling frames to retrieve information. Nodes may be sharing a single channel for sending data over to the sink or to another node. Simultaneous transmission of data on a single channel will lead to a collision, causing loss of data and energy. To avoid this, nodes should agree on a time slot at which a particular node would be sending. To agree on timeslots, nodes need to communicate, which requires a channel too. Considering the propagation delay, it is difficult for a node to know the instantaneous status of another node. The transceiver also consumes a large amount of energy while accessing the media. MAC controls activity of the transceiver to conserve energy [3].

\subsection{Energy Consumption Sources}

There are a few energy consumption sources at the MAC layer [4]:

Collision - when two or more nodes try to send information on a single channel at the same time, the packets collide. Collided packets need to be discarded and retransmitted.

Overhearing - when a node receives a packet destined for another node, it consumes unnecessary energy.

Overhead - sending and receiving control information also requires energy, causing an additional overhead.

Idle listening - idle listening is listening to an idle channel on which traffic is expected.

Over-emitting - sending information to a node which is not ready to receive. Hence, packets are discarded and need to be retransmitted.

\subsection{Performance Requirements for the MAC Layer}

While designing MAC layer protocols, one needs to consider the following requirements [5]:

Throughput: Protocol efficiency is measured by its throughput. In the case of a wireless link, it may be related to capacity.
Scalability: Scalability refers to the protocol's adaptation to an increase in network size, traffic, overhead and load. One way to deal with this is to localize the interactions so that nodes need less global knowledge to operate.

Latency: Latency can be referred as the time delay between message transmission and message arrival. Latency is an important constraint for time-critical applications, and needs to be minimized.

Number of hops: It is the number of hops taken by packets to reach the sink. Operation of the MAC protocol varies between single-hop and multi-hop scenarios. In the case of multiple hops taken to reach the sink, data needs to be aggregated before sending it to the sink.

\subsection{Classification of MAC Protocols}

MAC protocols can be categorized into two types [6]:

- schedule-based MAC protocol in which nodes agree upon a fixed schedule to access the channel. So, each node has a fixed slot for communication. Outside their slots, nodes move into sleep mode, avoiding collision and overhearing. The lifetime of nodes is enhanced, as they do not communicate over the complete duty cycle;

- random access-based protocol in which nodes need to compete to reserve access to a channel. After collision, each node waits for a random time before accessing the channel again. Energy efficiency of random access-based protocol is low.

\subsection{MAC Layer Protocols}

A protocol for an application can be chosen based on performance and specific requirements. In the battery-powered area Sensor-MAC, a T-MAC is presented. Then, MAC protocols based on energy harvesting are presented (Fig. 3).

Sensor-MAC (S-MAC): In general, nodes are synchronized locally, to operate a periodic sleep-and-listen schedule. Each node belongs to a virtual cluster and each cluster has a common listen-and-sleep schedule, as shown in Fig. 4. This represents the basic idea of S-MAC [7]. Each node discovers its neighbors regularly and establishes a link with them. Then, it assigns a distinct frequency, time or

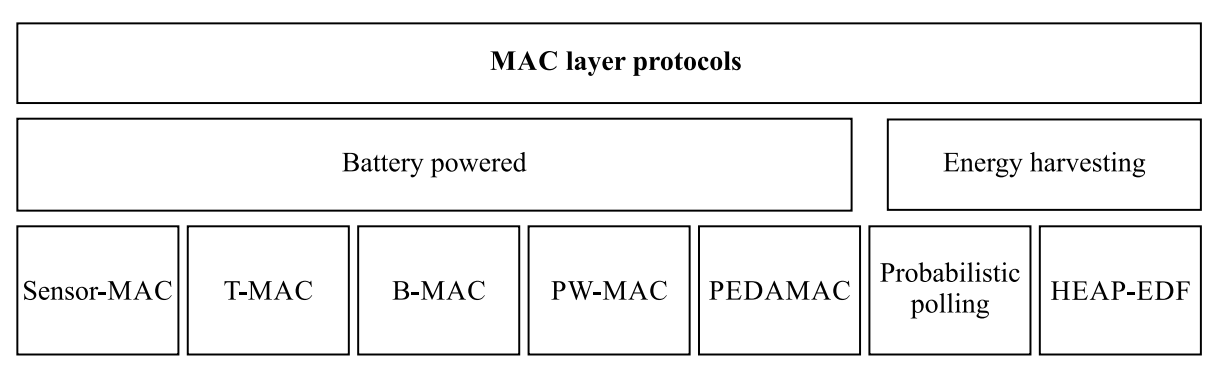

Fig. 3. MAC layer protocols. 
code to each link. Long messages are divided before sending. Such a solution offer various advantages, as it selforganizes the network to a variation in topology. This change in topology can be the consequence of deaths or movement of a node. It also operates a lower duty cycle, so the consumption of power used for overhearing and idle listening is reduced. Network latency increases as nodes alternate between active and sleep mode. It can be avoided altogether if a node wakes up after sensing the wake-up of its neighbor. Since sleep-and-listen periods are predefined, efficiency of the protocol may decrease under variable traffic, as traffic may be forwarded to a sleeping node.

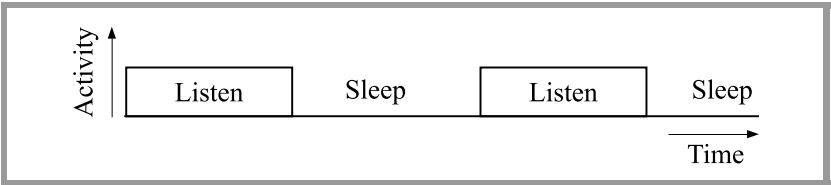

Fig. 4. Sleep-and-listen periods.

Timeout MAC (T-MAC): S-MAC has fixed listen and sleep periods, but applications with variable loads need dynamic listen and sleep periods. In T-MAC, the listen period ends when no event, such as receipt of data or sensing of activity has taken place for a threshold period (TP), as shown in Fig. 5 [8]. The listen period depends on current load. Transmission is based on Request-To-Send (RTS), Clear-To-Send (CTS) and acknowledgment (ACK) packets. Nodes close to the sink may have more data to send, so their listen periods are longer. The advantages are: RTS, CTS and ACK packets reduce collision rates and increase reliability. If listen periods are fixed, then nodes with less data will waste energy by idle listening. Energy consumption and idle listening are reduced as data can be sent in variable bursts. T-MAC has low sensitivity to latency, but it has a few drawbacks, such as it cannot support high data rate applications. Also, it has to trade-off throughput to maintain low energy consumption.

\section{$\stackrel{?}{2}$

\begin{tabular}{|c|c|c|c|c|}
\hline Listen & Sleep & Listen & Sleep & Listen \\
\hline
\end{tabular}

Time

Fig. 5. Adaptive listen and sleep periods.

Berkeley MAC (B-MAC): B-MAC uses preamble sampling [9], [10]. Each time a node wakes-up, it checks for any activity before sending. The node is also waiting only for a certain period of time to receive data. After timeout, the node returns to sleep mode. B-MAC uses clear channel assignment, and makes local policy decisions to optimize network performance. Owing to preamble sampling, the duty cycle is reduced, which increases efficiency and throughput. Energy consumption is lower because of low-power listening. Also, it supports reconfiguration to improve latency. B-MAC has a few drawbacks, such as it has no ability to handle multi-packet environments and suffers form a hidden terminal problem. Also, overhead of the protocol increases. The protocol can be enhanced further using adaptive preamble sampling.

Predictive Wake-up MAC (PW-MAC): In PW-MAC, the wake-up schedule of nodes can be randomized [11], [12]. To inform the intended transmitters, the node will send a signal upon waking up. A sender can predict the receiver's wake-up time and can wake-up simultaneously to save energy. To address timing challenges, PW-MAC has an on-demand prediction-based error correction mechanism. PW-MAC has a reduced duty cycle, as it has a random node wake-up schedule. It has improved performance compared to S-MAC and B- MAC, as collisions can be avoided. Latency is less than $5 \%$ of that typical of other MAC protocols. A node needs only 10 bytes of memory to store the prediction state of other nodes. Each node has to send a signal on waking-up, so the overhead of the protocol is increased, although it is low compared to other protocols. Also, hardware can induce errors in predicting wake-up times of the receiver.

Power Efficient and Delay Aware MAC (PEDAMAC): To minimize energy consumption due to overhearing, PEDAMAC transmits data at more than one power level. The access points (also called sinks) coordinate sensor nodes. Access points are assumed to have no power constraints, while sensor nodes have limited power. PEDAMAC assumes that each node can reach the sink in one hop. It has four phases: topology learning, topology collection, scheduling and adjustment. The protocol allows the nodes to operate at different power levels, as per the requirement of the task being processed by the node. It has three power levels: maximum power $P_{m}$, medium $P_{x}$, and minimum $P_{s}$. Synchronization is done at $P_{m}$. The sink can broadcast topology-related information at $P_{x}$. Data is transmitted at $P_{s}$. Low transmission power saves energy and it is used in delay-bound applications, but it has a few drawbacks, such as the fact that protocol assumes a one hop distance to the sink, which may not always be the case. Distinct power levels increase the protocol overhead. Also, data may be dropped before delivered, if transmission power is too low, i.e. the range of radio is decreased because of power limitation. PEDAMAC can be enhanced by increasing the number of media or channels to further reduce the delay [12]-[14].

Energy harvesting is considered as the only energy source by $\mathrm{Eu}$ et al. [15]. It is not easy to predict the wake-up schedule of nodes powered by energy harvesters. Authors exploited the uncertain nature of energy harvesting sources to increase the performance of MAC protocols. MAC protocols based on battery-powered WSNs have different goals, such as increased lifetime compared to energy harvesting based WSN (EH-WSN). So, there is a need to have protocols designed specifically for EH-WSN.

Probabilistic polling: In probabilistic polling, the sink sets contention probability $P_{c}$ in each node through a polling 
Table 1

Performance evaluation of MAC protocols

\begin{tabular}{|l|c|c|c|c|c|c|}
\hline Protocol & Throughput & $\begin{array}{c}\text { Energy } \\
\text { conservation }\end{array}$ & $\begin{array}{c}\text { Maximum \% of } \\
\text { energy saved } \\
\text { vs. S-MAC }\end{array}$ & Latency & Overhead & Scalability \\
\hline \hline S-MAC [7] & Low & Low & 0 & High & Low & High \\
\hline T-MAC [9] & Low & High & 85 & N/A & Moderate & Low \\
\hline B-MAC [9], [10] & High & Moderate & 57 & Moderate & High & Low \\
\hline PW-MAC [11], [12] & High & High & 80 & Low & Moderate & High \\
\hline PEDAMAC [13], [14] & Moderate & Moderate & 38 & Low & High & Low \\
\hline Probabilistic polling [15] & High & N/A & N/A & $\begin{array}{c}\text { Depends on energy } \\
\text { harvesting rate }\end{array}$ & Low & High \\
\hline HEAP-EDF [16] & Moderate & N/A & N/A & $\begin{array}{c}\text { Depends on energy } \\
\text { harvesting rate }\end{array}$ & Moderate & Low \\
\hline
\end{tabular}

packet [15]. Each node generates a random number $v$, and when it is less than contention probability $\left(v<P_{c}\right)$, the node is allowed to send. Otherwise, the node can go to the charging state. The sink keeps on changing contention probability depending on network response. If no sensor responds, the sink increases $P_{c}$. Also, when a node leaves the network, $P_{c}$ is increased. In the case of collision and joining of new node, $P_{c}$ is decreased by a larger amount. This approach is known as additive-increase and multiplicative-decrease. Contention probability $P_{c}$ offers maximum throughput when it is equal to the inverse of the number of nodes receiving polling packets:

$$
P_{o p t}=\frac{1}{N_{r}},
$$

where $P_{o p t}$ is the optimal probability that maximizes throughput. $N_{r}$ is the number of nodes receiving polling packets $\left(N_{r} \geq 1\right)$

This protocol can adapt to varying energy harvesting rates to ensure high throughput and the sink can also adjust $P_{c}$ in the case of a collision. Hence, the protocol increases scalability of the network. Since $P_{c}$ keeps changing due to collisions or when a node joins or leaves a network, it takes quite some time for the network to stabilize. This leads to increased network latency. Also, bandwidth is wasted until the network stabilizes at an appropriate $P_{c}$. Another drawback is that the protocol assumes a single hop distance to the sink, limiting protocol scalability.

HEAP-EDF: Power generated by ambient energy harvesting sources (HEAP), may vary, i.e. solar energy has different rates in the morning and in the afternoon. To overcome this, Earliest Deadline First (HEAP-EDF) uses a predictand-update algorithm to reduce the temporal variations [16]. In HEAP-EDF, the sink polls the node with the minimum or the earliest wake-up time. The sensor will not poll the node whose energy has decreased below the transmission level because of previous polling. At the powerbalance ratio of 0.5 , HEAP-EDF offers the best fairness.
The power-balance ratio is given as:

$$
\emptyset=\sum_{n=1}^{N} \frac{T_{c}}{T_{n}} .
$$

In Eq. (2), $T_{c}$ is the duration of polling cycle, $T_{n}$ is energy harvesting delay for $n$-th node and $N$ is the number of sensor nodes. Simulations in [16] show that channel utilization reduces as the link error probability increases. HEAP-EDF performs worse in the case of large networks. Also, the single-hop approach is assumed, which limits application of the protocol to small networks.

\subsection{Comparison of Protocols}

Table 1 shows the performance of MAC protocols reviewed. B-MAC has a high throughput owing to preamble sampling, but this increases the overhead too. Since probabilistic polling and HEAP-EDF are based on an ambient energy harvesting source, energy consumption is not a relevant factor to be compared. In this case of HEAP-EDF, overhead can be decreased if energy harvesting rates are correlated. Protocols with high overheads cannot be scaled to a large network due to the increase in the number of control

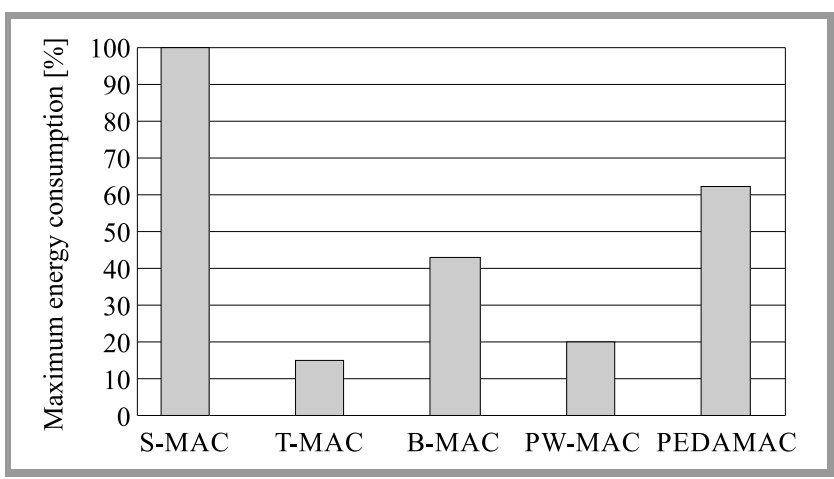

Fig. 6. Maximum energy consumption of protocols (considering S-MAC as a full-scale benchmark). 
packets. In PEDAMAC, as transmission power decreases, the range of radio also decreases, which affects the network scalability. Column 4 represents the relative percentage of energy saved. In the reviewed papers, simulations are performed under different scenarios and with different considerations, so it is difficult to directly compare these protocols. Hence, the comparison values are presented as percentages of S-MAC serving as a benchmark. Figure 6 shows the energy consumption analysis. S-MAC consumes $2.8 \mathrm{~mA} /$ node and T-MAC consumes $0.4 \mathrm{~mA} /$ node [8]. B-MAC saves 57\% more energy than S-MAC for a throughput of $240 \mathrm{~b} / \mathrm{s}$, because synchronization overhead increases in S-MAC [10]. PW-MAC protocol's duty cycle is only $11 \%$, while duty-cycle of S-MAC is $50 \%$ [11]. Decreased duty-cycle leads to decreased energy consumption. Also, owing to operation at distinct power levels, PEDAMAC saves 38\% more energy than S-MAC [14].

Table 2

Comparison of MAC protocols

\begin{tabular}{|c|c|c|c|}
\hline Protocol & Type & $\begin{array}{l}\text { Cross layer } \\
\text { optimiza- } \\
\text { tion }\end{array}$ & $\begin{array}{c}\text { Energy con- } \\
\text { servation } \\
\text { factors }\end{array}$ \\
\hline S-MAC [7] & $\begin{array}{c}\text { Single } \\
\text { hop }\end{array}$ & \multirow{7}{*}{ No } & $\begin{array}{l}\text { Overhearing, } \\
\text { idle listening }\end{array}$ \\
\hline T-MAC [8] & $\begin{array}{l}\text { Single } \\
\text { hop }\end{array}$ & & $\begin{array}{l}\text { Idle listening, } \\
\text { collision }\end{array}$ \\
\hline B-MAC [9], [10] & $\begin{array}{l}\text { Single } \\
\text { hop }\end{array}$ & & $\begin{array}{l}\text { Overhearing, } \\
\text { collision }\end{array}$ \\
\hline PW-MAC [11], [12] & $\begin{array}{l}\text { Multi } \\
\text { hop }\end{array}$ & & $\begin{array}{l}\text { Idle listening, } \\
\text { collision, } \\
\text { retransmission }\end{array}$ \\
\hline PEDAMAC [13], [14] & $\begin{array}{l}\text { Single } \\
\text { hop }\end{array}$ & & N/A \\
\hline $\begin{array}{l}\text { Probabilistic } \\
\text { polling [15] }\end{array}$ & $\begin{array}{l}\text { Single } \\
\text { hop }\end{array}$ & & N/A \\
\hline HEAP-EDF [16] & $\begin{array}{l}\text { Single } \\
\text { hop }\end{array}$ & & N/A \\
\hline
\end{tabular}

Table 2 shows the comparison of MAC protocols. Column 4 represents the factors that were focused on while designing the respective protocols, in order to reduce energy consumption. The key consideration of probabilistic polling and HEAP-EDF is the optimal use of harvested energy rather than conservation of energy.

\subsection{Open Research Problems}

A number of MAC protocols have been proposed and designed for WSN, but there are still many open issues that need to be addressed. Cross-layer interaction and optimization are potential areas of research which can enhance the performance of MAC protocols. The MAC protocols available can be analyzed for various traffic generation and node distribution models. Development of multi-hop MAC pro- tocols, in order to extend range and scalability, is another task to be considered in the future.

\section{Network Layer}

The main task of WSN is to sense and transmit data while using minimum resources. An efficient routing protocol is required at the network layer to choose a path with the minimum cost of delay, lifetime, energy or any other parameter that is more relevant to the application.

\subsection{Energy Consumption Sources}

Routing overhead is the main source of energy consumption at this particular level. Wang et al. presents one of the criteria to design the routing protocol with a minimum overhead to minimize energy consumption [17]. The overhead of a routing protocol varies with hop count and hop distance. In the case of small distances, single hop routing has less overhead. However, if the distance is long and cannot be covered with the available transmitted power, multi-hop routing is more efficient.

\subsection{Design Constraints of a Routing Protocol}

As compared to routing protocols designed for computer networks, WSN routing protocols need some distinctive features to handle a unique set of challenges [18], [19]:

Network scale: Node density may vary from hundreds to thousands, depending upon application. It is difficult to supervise such large, distributed structures. So, sensor nodes should be able to self-organize. The routing protocol should also deal with maintenance of global knowledge of such a large deployment.

Dynamics of node: WSNs are highly dynamic in nature. Owing to movement, power depletion and addition of new nodes, the topology of a WSN keeps changing. The routing protocol should be capable of adapting to frequent changes.

Resource constraints: A WSN need to operate on limited battery resources. Hence, the routing protocol should be able to transmit information over less than half a duty cycle. Some information-possessive applications need accuracy in data transmission. Therefore, the protocol should be able to trade-off energy consumption for accuracy.

Nature of node: Nodes operating over a certain coverage area may be homogenous or heterogeneous. Hence, the routing protocol needs to support nodes with unlike parameters and capabilities.

QoS: In a few applications delayed transmission of the sensed data may result in the loss of its significance. For such applications, delay is a critical parameter. Similarly, for a few other applications, other parameters - such as accuracy - may play a critical role. To maintain the quality of response of the application, these parameters need to be carefully traded-off for energy. 


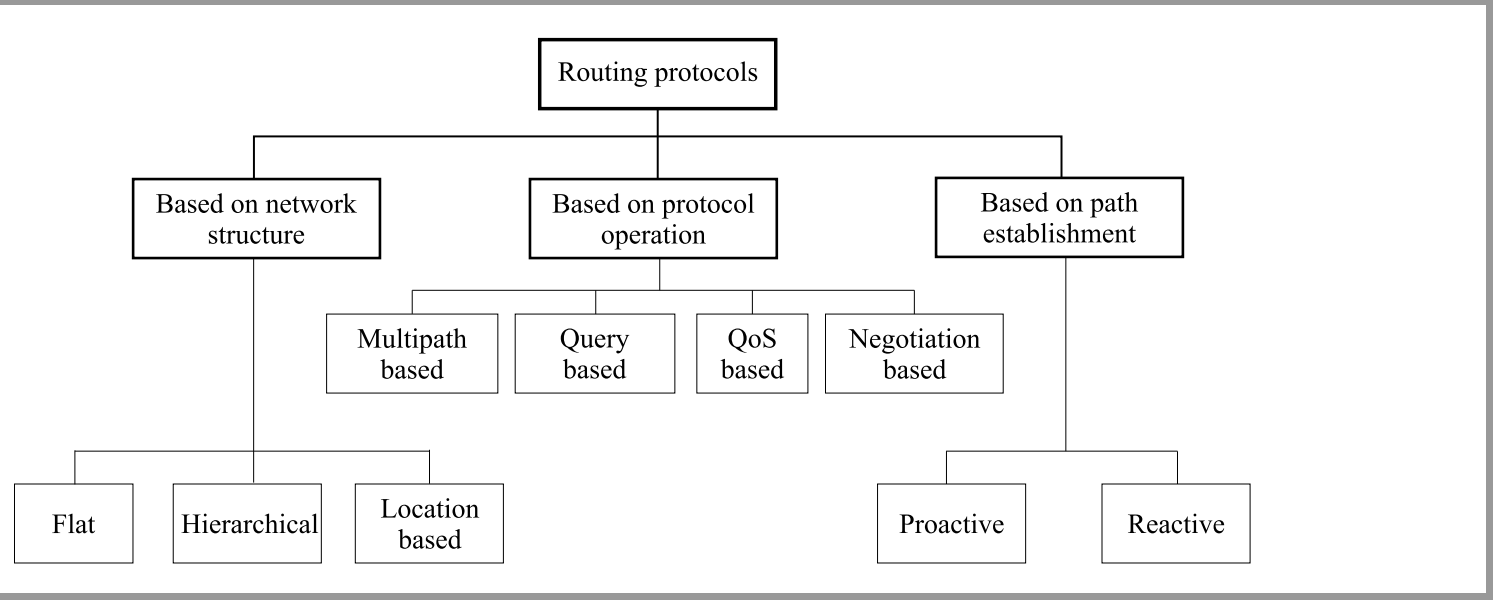

Fig. 7. Taxonomy of routing protocols.

\subsection{Classification of Routing Protocols}

Nodes can select from a number of available paths to transmit data to the sink or the base station. Routing protocols can be classified based on different criteria establishing the path to the sink, as shown in Fig. 7 [18], [20].

Routing protocols based on network structure:

Flat structure routing: All the nodes play the same role, i.e. each node is considered to be a base station and each node is provided with all information, so that the user can send a query to any node to get information.

Hierarchical structure routing: Not all nodes have the same capability. Higher capability nodes perform critical tasks, whereas less critical tasks are assigned to nodes with low capability. It is a two-level or multi-level structure.

Location-based routing: Nodes can be addressed based on their locations, whereas the location of a sensor can be detected using a satellite, provided the system is equipped with a low power GPS receiver. Another way is to measure the relative distance of the node from its neighbors based on strength of the signal received.

Routing protocols based on protocol operation:

Multipath routing protocol: In order to deliver data from source to destination, the protocol may rely on multiple paths. Multiple paths increase fault tolerance of the network, but also increase energy consumption and protocol overhead. An extension of the algorithm considers only the path with nodes having the highest energy. The path keeps changing whenever the protocol discovers a better path. By using the multipath routing protocol, reliability of the network can be increased in highly unreliable environments. A large packet can be divided into sub-packets and sent over different paths. A message can be reconstructed even if one of the sub-packets is lost due to link errors. Such an approach is known as multipath routing.

Query based routing: In query based routing, a node initiates a query and propagates it through the network. Each node receives the query and only the node having data that matches responds. Instead of propagating the query throughout the entire network, the node may send it in a random direction and wait for the response. If none of the nodes respond, then the node can propagate it through the whole network.

QoS based routing: In applications where parameters like delay, resources and bandwidth are critical, the routing protocol needs to maintain the quality and specifications of the critical parameter while delivering data. The routing protocol is responsible for maintaining a trade-off between energy and other metrics.

Negotiation based routing: Flooding and gossiping produce implosion and a single node may receive multiple data copies. The basic concept of the negotiation based protocol is to avoid propagation of duplicated packets. A sequence of negotiation messages is shared among the nodes to transmit redundant data to the next node. It reduces energy consumption and network congestion. The SPIN protocol discussed later is an example of the negotiation based protocol.

To deliver data from source to destination, the node initiating communication should know the path to the destination, i.e. path-based routing protocols are established in two ways:

Reactive path establishment: such protocols are eventdriven. After a data packet has reached a node, the protocol decides the next node to be taken towards the destination. The decision about the next node may depend upon cache history, but in most cases the nodes have limited memory and low computational capability, hence no cache history is maintained. Another metric to decide the next hop can include distance, cost, bandwidth and energy of the node.

Proactive path establishment: under this scenario, the protocol decides the path to destination when the data packet is at the first hop or at the node where communication initiates. The path can be established based on 

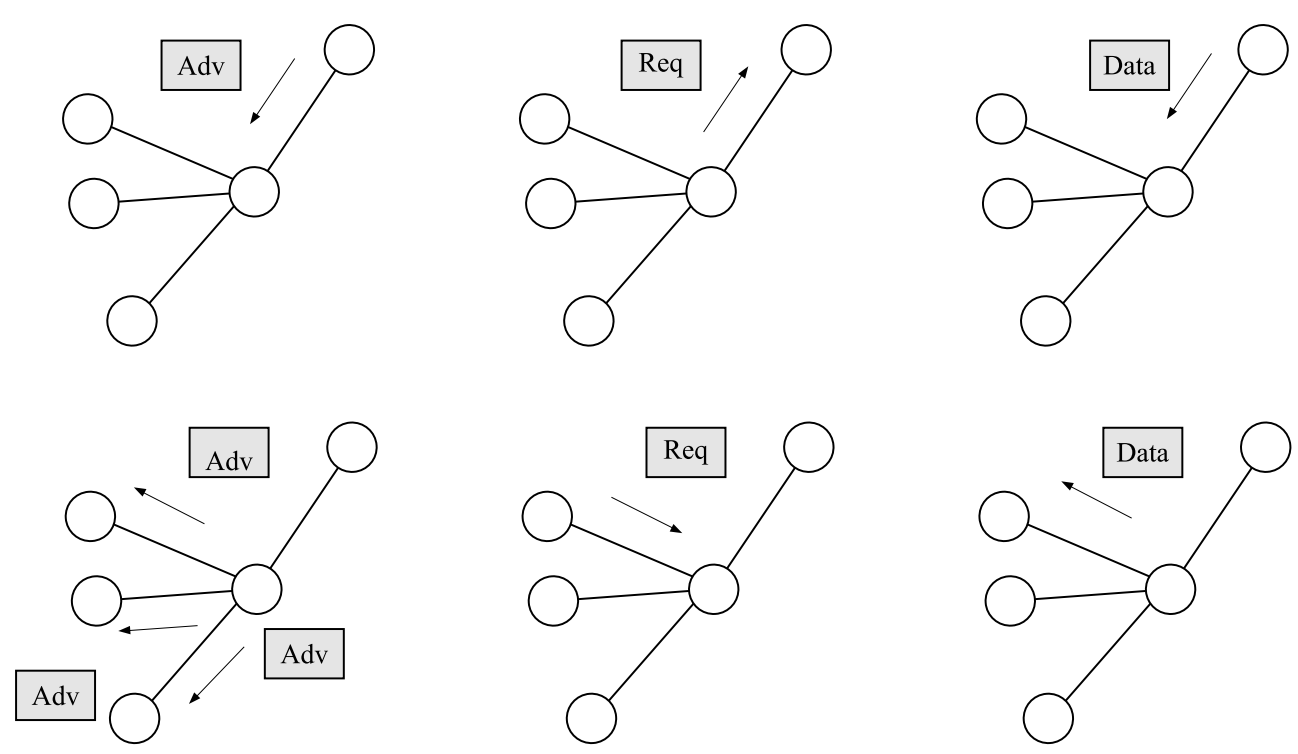

Fig. 8. SPIN protocol flowchart.

the minimum cost, maximum bandwidth or nodes with the highest energy levels. Once the path is established, all data packets propagate through the path selected. These protocols are not fault-tolerant, as data will be lost if the established link fails.

\subsection{Network Layer Protocols}

Flooding and gossiping: Flooding allows a node to send packets via all links. To avoid a packet looping indefinitely, the hop count or time-to-live is included in the packet. Another approach is gossiping, in which the packet is not sent via all outward links. The packet is transmitted only to a randomly selected neighbor. This saves bandwidth of the network but increases the delay from source to destination [21].

Sensor Protocols for Information via Negotiation (SPIN): SPIN overcomes the drawbacks of conventional dissemination protocols. SPIN is based on metadata [22]. Transmitter broadcasts the metadata of data. The receiver checks the information about data and sends the request to the transmitter if interested. Finally, the transmitter transmits the information to the interested receiver.

The SPIN protocol is presented in Fig. 9, where Adv are advertisement packets advertising metadata, Req are request for data packets from interested nodes to transmitter and Data are packets carrying data.

Directed diffusion: In directed diffusion, data packets are propagated through the network as interests, whereas the reverse reply link towards the transmitter is known as gradient [23]. Each node maintains a cache. When an event occurs, the node searches its cache. If the entry is not in the cache, it is added for future use. Caching increases efficiency and decreases energy consumption. Using the sequence of interests and gradients, the best path is reinforced between the transmitter and the receiver. Directed diffusion is based on the localized demand-driven query model. Receiver queries the sender node through interests for data and gets the response. The query-driven model increases the overhead.

Low-Energy Adaptive Clustering Hierarchy (LEACH): LEACH is a hierarchical cluster-based protocol. Nodes with higher energy are cluster heads [24] collecting information from all nodes in the cluster. Aggregated data is compressed and sent to the sink. LEACH reduces energy consumption, because cluster heads can be selected efficiently to increase network lifetime. The node generates a random number between 0 and 1 , if the number generated is less than $T(n)$, the node can become a cluster head. The threshold ensures that the node has not become the cluster head in last $\frac{1}{p}$ rounds:

$$
T(n)= \begin{cases}0 & \text { if } n \notin G \\ \frac{p}{1-p\left(r \bmod \left(\frac{1}{p}\right)\right)} & \forall n \in G,\end{cases}
$$

where $T(n)$ is the threshold to choose the cluster head, $G$ is the set of all nodes eligible for cluster head role, $p$ is probability of being the cluster head and $r$ is the current round number.

LEACH protocol is demonstrated in Fig. 9, where sensor nodes send data to cluster heads and cluster heads send aggregated data to the base station.

LEACH with Spare Management (LEACH-SM): It is a modification of the LEACH protocol. LEACH-SM has spare nodes which are normally in the sleep mode [25]. 
Table 3

Performance evaluation of MAC protocols

\begin{tabular}{|l|c|c|c|c|c|}
\hline \multicolumn{1}{|c|}{ Protocol } & Type & $\begin{array}{c}\text { Energy } \\
\text { consumption }\end{array}$ & Network lifetime & Mobility & Scalability \\
\hline \hline Flooding and gossiping [21] & Flat & High & Small & Yes & Low \\
\hline SPIN [22], [28] & Negotiation based & Low & Small & Yes & Low \\
\hline Directed diffusion [23] & Multipath & Moderate & Small & Limited & Low \\
\hline LEACH [24], [29] & Hierarchical & High & Medium & No & Moderate \\
\hline LEACH-SM [25] & Hierarchical & Moderate & Long & No & Moderate \\
\hline DEEC [26] & $\begin{array}{c}\text { Hierarchical, multilevel, } \\
\text { heterogeneous }\end{array}$ & Low & Long & No & High \\
\hline BLR [27], [28] & Location based & Low & Moderate & Limited & High \\
\hline
\end{tabular}

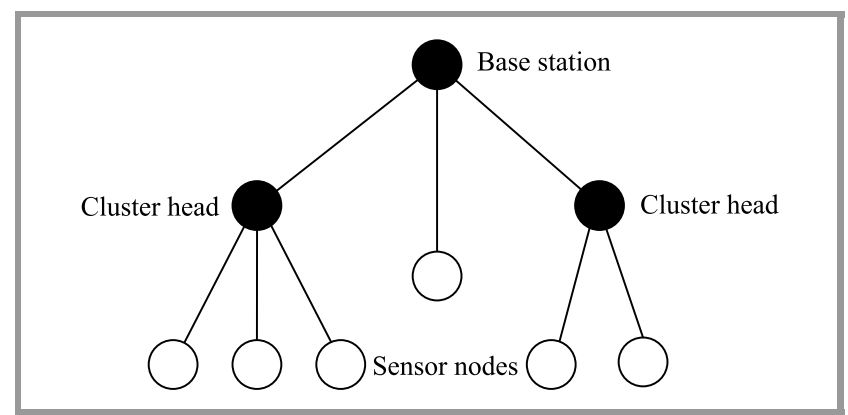

Fig. 9. Structure of a LEACH protocol network.

When the network is out of energy, spare nodes provide redundancy and increase network lifetime. LEACH-SM also has the capability to avoid deadlocks that may occur due to redundancy of nodes, and thus offers extended lifetime.

Distributed Energy Efficient Clustering (DEEC): DEEC was proposed for heterogeneous WSNs [26]. It considers multi-level heterogeneity. Other clustering protocols did not consider energy while choosing cluster heads. DEEC uses the knowledge about initial and residual energy of nodes while choosing cluster heads. DEEC used the same threshold in Eq. (3) to determine the cluster head, but threshold probability to select the cluster head depends on the heterogeneity of nodes.

$$
p=\left\{\begin{array}{ll}
\frac{p^{\prime} E(r)}{(1+a m) E^{\prime}(r)} & \text { if node is normal } \\
\frac{p^{\prime}(1+a) E(r)}{(1+a m) E^{\prime}(r)} & \text { if node is advanced }
\end{array},\right.
$$

where $p^{\prime}$ is reference probability, $E(r)$ is residual energy and $E^{\prime}(r)$ is average energy of the network, $m$ is fraction of advanced nodes whose energy is $a$ times higher than that of normal nodes. Normally, a cluster dies as its cluster head is out of energy. DEEC keeps on reassigning the role of the cluster head depending upon energy, to extend the lifetime of network. The DEEC stability period is $15 \%$ longer than in the stable election protocol. Also, it does not require any global knowledge to select the cluster head. Hence, it is more efficient than other clustering protocols.

Beacon-less Routing (BLR): In location based routing, nodes exchange a few messages called beacons to know the position of each other. These beacons create a large overhead and work inefficiently in erroneous wireless links. Therefore, BLR was proposed. BLR selects the next hop by computing the dynamic forwarding delay. A node broadcasts a data packet to all its neighbors but only the receiving node which is best positioned towards the destination, will forward the packet. Nodes within a certain area take part in forwarding. These areas are called forwarding areas and can be of any shape. The receiving node sends a passive acknowledgment back to the sending node [27], [28].

\subsection{Comparison Table}

Table 3 shows the comparison of the protocols' performance. Since the SPIN protocol is based on metadata, its energy consumption is low. The BLR protocol has a lower overhead. Hence, it is highly scalable and can be used for large networks. Figure 10 represents a lifetime analysis of the hierarchical protocols reviewed. The lifetime of LEACH-SM equals $183 \%$ of the lifetime of LEACH [25]. Also the lifetime of DEEC is $130 \%$ of the lifetime of LEACH [26].

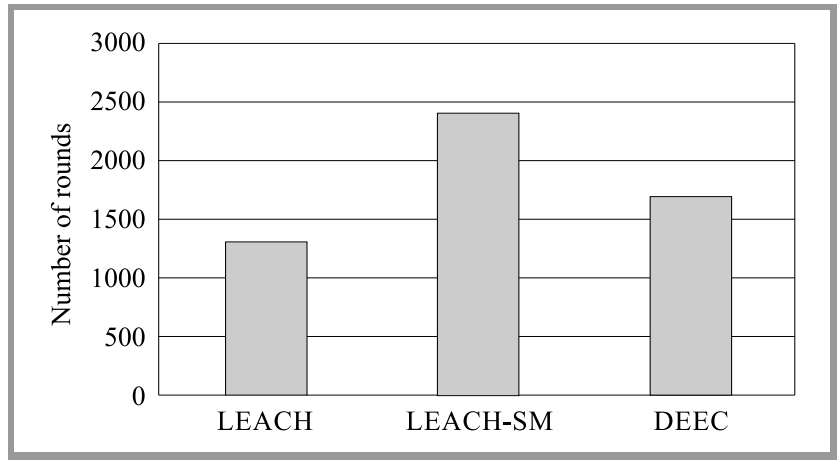

Fig. 10. Lifetime of hierarchical protocols. 


\subsection{Open Research Problems}

Most routing protocols assume a channel to be loss-less. So, future work includes development of routing protocols for lossy wireless channels. Also, existing routing protocols can be evaluated for lossy channels. QoS can be enhanced to ensure latency-free routing. Security management can be explored to avoid such threats as sleep deprivation attacks, packet dropping attacks and collecting sensitive information [30]. The routing protocols considered can be upgraded to handle various types of traffic, and the effect of traffic on the lifetime of a network can be minimized. Different traffic profiles should be modeled and analyzed to design efficient routing protocols [31]. New routing protocols are required for mobile networks. Also, most routing protocols available assume that an ideal MAC protocol exists. Cross-layer optimization can be used to improve the performance of a network as a whole.

\section{Cross Layer Approach}

To improve network performance, interaction of parameters across the protocol stack is necessary. Energy is a parameter of the physical layer and routing is considered at the network layer. Layers need to interact to obtain the value of energy in a routing packet. This helps the routing protocol to choose an energy efficient path. Route energy packets which are used to exchange energy values among nodes are generated using the cross-layer design. Hoesel et al. presents a cross-layer approach in which the routing protocol uses topology and infrastructure information available at the MAC layer [32]. It reestablishes the route utilizing information at the MAC layer and outperforms S-MAC and Dynamic Source Routing (DSR) in mobile sensor networks [33]. Cross Layer MAC (CL-MAC) makes and optimizes scheduling decisions based on cross layer information [34]. Path-Loss Ordered Slotted Aloha (PLOSA) protocol is designed using cross-layer design for wireless data collection networks [35]. It helps in observing physical signals and orders the access of nodes accordingly. Nodes at a greater distance from the collector get an earlier chance to access the slot of the transmission channel. PLOSA has a high delivery rate and low latency.

A cross-layer approach has been presented by Catarinucci et al., in which protocol solutions are integrated with hardware [36]. A new wake-up system consisting of a sensor node and a power meter circuit was suggested in the paper, where the suggested protocol exploits the hardware to reduce power consumption. It is indicated by Alrajeh et al. that to design a secure routing protocol, the crosslayer approach was necessary [37]. Most security attacks are multilayered. The sleep deprivation attack, for instance, occurs at the physical layer, whereas the packet dropping attack occurs at the network layer. The author proposed to keep an eye on the packet count, in order to prevent malicious nodes from sending unnecessary packets and creating congestion. Hence, in order to select an energy efficient and secure path, cross-layer communication is necessary.

\section{Conclusions and Future Works}

In this paper existing MAC protocols and routing protocols have been surveyed. MAC protocols have been reviewed for both type of nodes followed by their advantages and disadvantages. This paper suggests that because of its low latency, PEDAMAC can be used for delay sensitive applications. Owing to the random wake-up schedule, PW-MAC offers high throughput. It sends one update in $1400 \mathrm{~s}$, so overhead is moderate.

Diversified routing protocols ranging from flat to multilevel have been discussed in this paper. This paper analyses that DEEC has 30\% more rounds than LEACH, because of low energy consumption. LEACH-SM has $83 \%$ more rounds than LEACH. Then, the cross-layer approach has been presented that improves performance of the protocol stack as a system. Although the protocols discussed may seem promising, there are still many challenges that need to be faced in WSNs. The cross-layer approach is a research area that needs to be studied and analyzed widely. Traffic modeling is another prospective area which can be analyzed and studied for improving performance or security of networks. Energy harvesting algorithms and models for WSNs also are subject to great advancements in the future.

\section{References}

[1] M. Abozahhad, M. Farrag, and A. Ali, "A comparative study of energy consumption sources for wireless sensor networks", Int. J. of Grid Distrib. Comput., vol. 8, no. 3, pp. 65-76, 2015.

[2] J. Zheng and A. Jamalipour, Wireless Sensor Networks: A Networking Perspective. Wiley, 2014.

[3] A. S. Althobaiti and M. Abdullah, "Medium access control protocols for wireless sensor networks classifications and cross-layering", Procedia Comp. Sci., vol. 65, pp. 4-16, 2015.

[4] W. Ye, J. Heidemann, and D. Estrin, "An energy-efficient MAC protocol for wireless sensor networks", in Proc. of 21st Ann. Joint Conf. of the IEEE Comp. and Commun. Soc. IEEE INFOCOM 2002, New York, NY, USA, 2002, pp. 1567-1576.

[5] K. Sohraby, D. Minoli, and T. Znati, Wireless Sensor Networks Technology, Protocols and Applications, 2nd ed. Wiley, 2013.

[6] U. Roedig, "f-MAC: A deterministic media access control protocol without time synchronization", in Wireless Sensor Networks Third European Workshop, EWSN 2006 Zurich, Switzerland, February 13-15, 2006 Proceedings, K. Römer, H. Karl, and F. Mattern, Eds., LNCS 3868. Zurich: Springer, 2006, pp. 276-291.

[7] M. A. Ameen, S. M. R. Islam, and K. Kwak, "Energy saving mechanisms for MAC protocols in wireless sensor networks", Int. J. of Distrib. Sensor Netw., vol. 6, no. 1, Article ID 163413, 2010 (doi: 101155/2010/163413).

[8] L. Wang and K. Liu, "An adaptive energy-efficient and low-latency MAC protocol for wireless sensor networks", in Proc. Int. Conf. on Wirel. Commun., Netw. and Mob. Comput. WiCOM 2007, Shanghai, China, 2007, pp. 2440-2443.

[9] C. Cano, B. Bellalta, A. Sfairopoulou, and M. Oliver, "Low energy operation in WSNs: A survey of preamble sampling MAC protocols", Comp. Netw., vol. 55, no. 15, pp. 3351-3363, 2011.

[10] J. Polastre, J. Hill, and D. Culler, "Versatile low power media access for wireless sensor networks", in Proc. 2nd Int. Conf. on Embed. Netw. Sensor Syst. SenSys 2004, Baltimore, MD, USA, 2004. 
[11] D. B. Johnson, "PW-MAC: An energy-efficient predictive-wakeup MAC protocol for wireless sensor networks", in Proc. 30th IEEE Int. Conf. on Comp. Commun. IEEE Infocom 2011, Shanghai, China, 2011, pp. 1305-1313.

[12] J. Kabara and M. Calle, "MAC protocols used by wireless sensor networks and a general method of performance evaluation", Int. J. of Distrib. Sensor Netw., vol. 8, no. 1, 2012 (doi: 10.1155/2012/834784).

[13] S. Coleri and P. Varaiya, "PEDAMACS: Power efficient and delay aware medium access protocol for sensor networks", IEEE Trans. on Mob. Comput., vol. 5, no. 7, pp. 920-930, 2006.

[14] M. G. C. Torres, "Energy consumption in wireless sensor networks using GSP", Master's Thesis, University of Pittsburgh, 2006.

[15] Z. A. Eu, H. P. Tan, and W. K. G. Seah, "Design and performance analysis of MAC schemes for Wireless Sensor Networks Powered by Ambient Energy Harvesting", Ad Hoc Netw., vol. 9, no. 3, pp. 300-323, 2011.

[16] Y. Jin and H. P. Tan, "Optimal performance trade-offs in MAC for wireless sensor networks powered by heterogeneous ambient energy harvesting", in Proc. IEEE IFIP Networking Conf. IFIP Networking 2014, Trondheim, Norway, 2014 (doi: 10.1109/IFIPNetworking.2014.6857125).

[17] Q. Wang, M. Hempstead, and W. Yang, "A realistic power consumption model for wireless sensor network devices", in Proc. 3rd Ann. IEEE Commun. Soc. on Sensor and Ad Hoc Commun. and Netw. SECON 2006, Reston, VA, USA, 2006, pp. 286-295 (doi: 10.1109/SAHCN.2006.288433).

[18] J. N. Al-Karaki and A. E. Kamal, "Routing techniques in wireless sensor networks: A survey”, IEEE Wirel. Commun., vol. 11, no. 6, pp. 6-28, 2004 (doi: 10.1109/MWC.2004.1368893).

[19] K. Akkaya and M. Younis, "A survey on routing protocols for wireless sensor networks", Ad Hoc Netw., vol. 3, no. 3, pp. 325-349, 2005.

[20] S. P. Singh and S. C. Sharma, "A survey on cluster based routing protocols in wireless sensor networks", Procedia Comp. Sci., vol. 45, no. C, pp. 687-695, 2015.

[21] I. F. Akyildiz, W. Su, Y. Sankarasubramaniam, and E. Cayirci, "Wireless sensor networks: A survey", Comp. Netw., vol. 38, no. 4, pp. 393-422, 2002.

[22] W. R. Heinzelman, J. Kulik, and H. Balakrishnan, "Adaptive protocols for information dissemination in wireless sensor networks", in Proc. 5th Ann. ACM/IEEE Conf. on Mob. Comput. and Netw. MobiCom'99, Seattle, WA, USA, 1999, pp. 174-85.

[23] C. Intanagonwiwat, R. Govindan, and D. Estrin, "Directed diffusion: A scalable and robust communication", in Proc. 6th Ann. Int. Conf. on Mob. Comput. and Netw. MobiCom'00, Boston, MA, USA, 2000, pp. 56-67.

[24] M. M. Afsar and M. H. Tayarani-N, "Clustering in sensor networks: A literature survey", J. of Netw. and Comp. Appl., vol. 46, pp. 198-226, 2014.

[25] B. A. Bakr and L. T. Lilien, "Comparison by simulation of energy consumption and WSN lifetime for LEACH and LEACH-SM", Procedia Comp. Sci., vol. 34, pp. 180-187, 2014.

[26] L. Qing, Q. Zhu, and M. Wang, "Design of a distributed energyefficient clustering algorithm for heterogeneous wireless sensor networks", Comp. Commun., vol. 29, no. 12, pp. 2230-2237, 2006.

[27] J. A. Sanchez, P. M. Ruiz, and R. Marin-Perez, "Beacon-less geographic routing made practical: Challenges, design guidelines, and protocols", IEEE Commun. Mag., vol. 47, no. 8, pp. 85-91, 2009.

[28] M. Heissenbüttel, T. Braun, T. Bernoulli, and M. Wälchli, "BLR: Beacon-less routing algorithm for mobile ad hoc networks", Comp. Commun., vol. 27, no. 11, pp. 1076-1086, 2004.

[29] L. J. G. Villalba, A. L. S. Orozco, A. T. Cabrera, and C. J. B. Abbas, "Routing protocols in wireless sensor networks", Sensors, vol. 9, no. 11, pp. 8399-8421, 2009.

[30] K. Chelli, "Security issues in wireless sensor networks: Attacks and countermeasures", in Proc. of the World Congr. on Engin. WCE 2015, London, United Kingdom, 2015, vol. I, pp. 519-524.
[31] Q. Wang, "Traffic analysis and modeling in wireless sensor networks and their applications on network optimization and anomaly detection", Netw. Prot. and Algorithms, vol. 2, no. 1, pp. 74-92, 2010 (doi: 10.5296/npa.v2i1.328).

[32] L. Van Hoesel, T. Nieberg, J. Wu, and P. J. M. Havinga, "Prolonging the lifetime of wireless sensor networks by cross-layer interaction", IEEE Wirel. Commun., vol. 11, no. 6, pp. 78-86, 2004.

[33] D. B. Johnson and D. A. Maltz, "Dynamic source routing in ad hoc wireless networks", Mob. Comput., vol. 353, pp. 153-181, 1996.

[34] M. S. Hefeida, T. Canli, and A. Khokhar, "CL-MAC: A cross-layer MAC protocol for heterogeneous wireless sensor networks", Ad Hoc Netw., vol. 11, no. 1, pp. 213-225, 2013.

[35] D. Espes, X. Lagrange, and L. Suárez, "A cross-layer MAC and routing protocol based on slotted aloha for wireless sensor networks", Annales des Telecommun./Annals of Telecommun., vol. 70, no. 3-4, pp. 159-169, 2015.

[36] L. Catarinucci et al., "A cross-layer approach to minimize the energy consumption in wireless sensor networks", Int. J. of Distrib. Sensor Netw., vol. 10, no. 1, 2014 (doi: 10.1155/2014/268284).

[37] N. A. Alrajeh, J. Lloret, and J. Loo, "Secure routing protocol using cross-layer design and energy harvesting in wireless sensor networks", Int. J. of Distrib. Sensor Netw., vol. 9, no. 1, 2013 (doi: $10.1155 / 2013 / 374796)$.

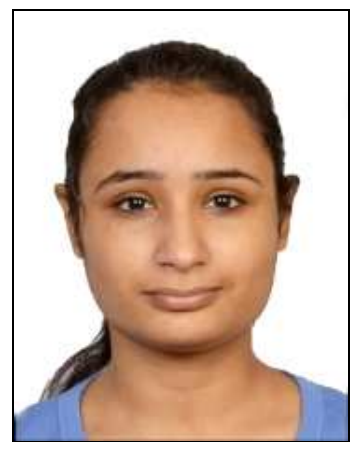

Aarti Kochhar is working as an Assistant Professor at Lovely Professional University, Phagwara, India. She has completed her M.E. degree in Electronics and Communication Engineering. She conducts research in the areas of wireless sensor networks.

E-mail: aarti.kochhar92@gmail.com

University Institute of Engineering and Technology Panjab University

Chandigarh, India

Lovely Professional University

Phagwara, India

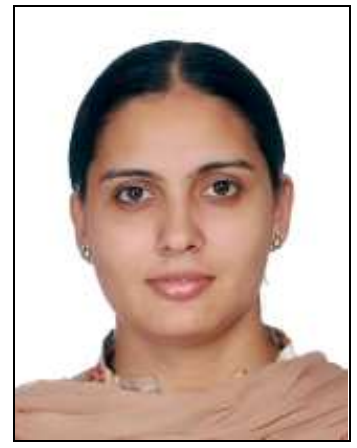

Pardeep Kaur is working as an Assistant Professor at the Electronics and Communication Engineering Department at U.I.E.T., Panjab University, Chandigarh, India. She received her B.Tech. and M.E. degrees in Electronics and Communication Engineering. She is pursuing her Ph.D. in wireless sensor networks. Her areas of interest include optical communication and wireless communication.

E-mail: pardeep.tur@gmail.com

University Institute of Engineering and Technology

Panjab University

Chandigarh, India 


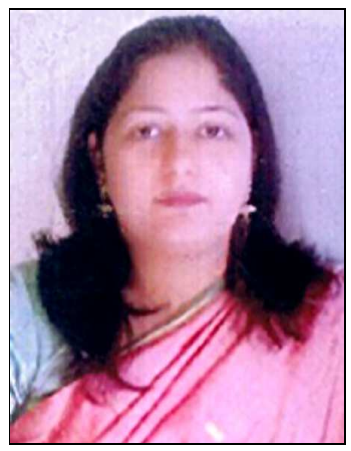

Sukesha Sharma is working as an Assistant Professor at the Electronics \& Communication Engineering Department at U.I.E.T., Panjab University, Chandigarh, India. She has completed her B.Tech. and M.E. degrees in Electronics and Communication Engineering. Her research interests include embedded systems, automation and control, active vibration control and energy harvesting.

E-mail: er_sukesha@yahoo.com

University Institute of Engineering and Technology Panjab University

Chandigarh, India

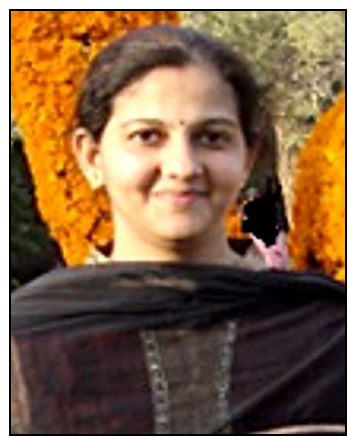

Preeti Singh is working as an Assistant Professor at the Electronics \& Communication Engineering Department at U.I.E.T., Panjab University, Chandigarh, India. She has completed her B.Tech. and M.E. degrees in Electronics and Communication Engineering. She obtained her Ph.D. degree in 2013. Her areas of interest include optical communication (wired and wireless), optical biosensors and cognitive neuroscience.

E-mail: preets.singh.82@gmail.com

University Institute of Engineering and Technology Panjab University

Chandigarh, India 